\title{
Alpha-Tocopherol and Cyanocobalamin Combination Accelerates Peripheral Nerve Healing: An Experimental Animal Study
}

\author{
Cem ALBAY ${ }^{1}$, Asli KAHRAMAN AKKALP² \\ ${ }^{1}$ Metin Sabancı Baltalimanı Bone Diseases Research and Training Hospital, Department of Orthopaedics and Traumatology, Istanbul, Turkey \\ ${ }^{2}$ Katip Celebi University, Ataturk Training and Research Hospital, Department of Pathology, Izmir, Turkey
}

Corresponding author: Cem ALBAY cemalbay@hotmail.com

\section{ABSTRACT}

AIM: To evaluate the functional and histopathological results of alpha-tocopherol (vitamin $\mathrm{E}$ ) and vitamin $\mathrm{B}_{12}$ on an experimental rat model of peripheral nerve injury.

MATERIAL and METHODS: This research included 32 Wistar Hannover rats. The sciatic nerves of the animals were crushed using an aneurysm clamp. The rats were divided into 4 groups, as group 0 (the controls; no treatment), and groups $B_{12}$, $E$, and $B_{12}+E$, respectively. The rats were analyzed functionally, using the sciatic functional index (SFI), and histopathologically.

RESULTS: In the functional analysis, it was determined that vitamin $\mathrm{E}$ was as influential as $\mathrm{B}_{12}$. Concomitant use of these 2 vitamins was found to be more beneficial. The SFI was significantly higher in the $\mathrm{B}_{12}+\mathrm{E}$ group when compared with that of the B12 group, which indicated that vitamin $E$ improved the healing effects of vitamin $B_{12}$. In the histopathological evaluation, vitamin $E$ was not effective in the treatment of axonal degeneration ( $A x D$ ) or edema/inflammation (El) by itself. Although vitamin $B_{12}$ was effective in the treatment of El, it was ineffective in the treatment of AxD. However, the combination of these vitamins decreased both AxD and El, which showed that the additive effects of these vitamins could reverse neurological injury when used together.

CONCLUSION: Vitamins $B_{12}$ and $E$ were effective in the functional recovery of peripheral nerve injury (PNI). Neither vitamin $B_{12}$ nor $E$ was effective in the treatment $A x D$; however, their combination was effective in the treatment of $A x D$. The results suggested that vitamin $\mathrm{E}$ was effective in the treatment of $\mathrm{PNI}$, especially when combined with vitamin $\mathrm{B}_{12}$. It is our belief that the combination of these vitamins could be used in the treatment of PNI, especially after future studies have been conducted on humans.

KEYWORDS: Alpha-tocopherol, Axonal degeneration, Cyanocobalamin, Peripheral nerve injury, Sciatic nerve, Rats

ABBREVIATIONS: PN: peripheral nerve, PNI: PN injury, AxD: Axonal degeneration, AL: Axonolysis (vacuolization), El: Edema/ inflammation, PNR: PN regeneration, PNS: Peripheral nervous system, CNS: Central nervous system, B12: Cyanocobalamin, E: Alpha-tocopherol, SN: Sciatic nerve, SFI: Sciatic functional index, WTA: Walking tract analysis, RoS: Reactive oxygen species, NGF: Nerve growth factors.

\section{INTRODUCTION}

rushing, cutting, or stretching injuries of the peripheral nerve $(\mathrm{PN})$ are the main mechanisms that can cause PN injury (PNI) (17). As PNI is seen mostly in younger patients with a mean age of 38.1 years old, it is an important cause of functional, social, and psychological problems and economic loss $(12,24)$. PNI can induce complex pathophysiological processes, including edema/inflammation (EI), demyelination, axonolysis (AL) and/or axonal degeneration (AxD) of the nerve, which may result in exacerbated damage 
mechanisms or repair mechanisms of the nerve tissue (15). Although exacerbated damage of the PN can cause severe sensorial and/or motor dysfunction, $\mathrm{PN}$ regeneration (PNR) is possible because of the repair mechanisms in the peripheral nervous system (PNS).

Surgical techniques have been improved over recent decades. Even though the outcomes have not been sufficiently good, surgical intervention is still accepted as the main treatment for complete transections of the PN (3). However, PNR is believed to be possible for incomplete PNI, which is usually treated with medicines such as thymoquinone, alpha lipoid acid, omega 3-6, and several vitamins $(1,6)$.

The number of studies about the healing effects of vitamin supplementations on PNIs has increased over recent years. Most of these studies have been about cyanocobalamin (vitamin $\mathrm{B}_{12}$ ), which is the most widely known and recommended medical supplementation for positive regeneration effects with neurotrophic factors, which stimulate the regeneration of both the myelin and axons $(1,2)$. Alpha-tocopherol (vitamin E) is a chain-breaking antioxidant. It ensures membranous solidity (23). Vitamin E has been shown to be neuroprotective in patients with diabetes, alcohol abuse, and cisplatin usege (9). In addition, vitamin E deficiency by itself is known to be a cause of peripheral neuropathy. However, the effects of vitamin $\mathrm{E}$ on nerve regeneration after $\mathrm{PNI}$ is not yet as clear as those of vitamin $\mathrm{B}_{12}$.

The purpose herein was to present gait analysis outcomes and histological results of vitamins $E$ and $B_{12}$ alone and in combination.

\section{- MATERIAL and METHODS}

\section{Subjects}

The study animals comprised 32 Wistar Hannover rats, weighing 200 to $250 \mathrm{~g}$, at the Experimental Research Center of the. The study began after it was determined to be feasible through a preliminary study using 2 rats. The animals were randomly divided into 4 groups with 8 rats in each group, as determined using standard power analysis. The rats were weighed daily for dose evaluation. The temperature of the room and humidity were maintained at $22{ }^{\circ} \mathrm{C}$ and $50 \%$, respectively. The study was conducted for 28 days.

\section{Surgery}

It was decided that damage the sciatic nerve (SN) would be performed as the PNI, as it has both motor and sensorial functions. An intraperitoneal mixture of $70 \mathrm{mg} / \mathrm{kg}$ of ketamine hydrochloride and $10 \mathrm{mg} / \mathrm{kg}$ of xylazine hydrochloride was used for the anesthetics. Surgery was performed via a 1-inch incision following subcutaneous tissue dissection, just below the left iliac crest, 1-cm lateral to the middle line, and extending to the popliteal fossa. The left SN and embranchment were completely exposed by dissecting the superficial gluteal muscle and biceps femoris, and the fascia surrounding the junction line of the surface. Next, an area of the nerve $10 \mathrm{~mm}$ above the embranchment was damaged using a clamp. The same clamp was used for all of the rats and maintained for
$15 \mathrm{~min}$. Subcutaneous $0.1 \mathrm{cc} / \mathrm{kg}$ carprofen was used for pain control of the rats immediately after closing the incision. All of the procedures were standardized and applied by the first author.

Medication was started just after completion of the crush injury. The vitamin doses were determined using those of previous studies in corporation with veterinarians $(26,28)$. The treatments were applied to all of the rats at the same time each day for 4 weeks. The crush injury severity and period of damage were homogenized by applying the same $50-\mathrm{g}$ closing pressure clamp to all of the rats for the same period of time. All of the rats were observed individually until the incision closure. Next, the rats were combined and 8 rats were placed into each cage. They were not subjected to postoperative restrictions for movement or diet. No local or systemic complications were observed.

\section{Groups of the Subjects and Treatment Doses}

According to the sample size determination using the standard power analysis, 4 groups were established, with 8 rats per group, as given below.

\section{Group 0: control (no treatment)}

Group $B_{12}: 1 \mathrm{mg} / \mathrm{kg}$ intraperitoneal cyanocobalamin for 28 days

Group E: 100 mg/kg intraperitoneal Alpha-tocopherol for 28 days

Group $\mathrm{B}_{12}+\mathrm{E}: 1 \mathrm{mg} / \mathrm{kg}$ intraperitoneal cyanocobalamin and $100 \mathrm{mg} / \mathrm{kg}$ Alpha-tocopherol for 28 days

The treatment dose was calculated according to the weights of the rats each day during the study protocol.

\section{Functional Evaluation}

Following the completion of medication process at the end of the 28 days, walking tract analysis (WTA) was performed. Functional outcomes were evaluated using sciatic functional index (SFI) measurements.

The most important measurement of nerve healing is functional recovery. Electromyography (EMG) may not show a connection between functional improvement and the healing date (15).

An axon can grow approximately 1-3 mm per day (7). Therefore, the SFI, which is measured by the WTA, is one of the most widely used and valuable assessment methods.

The SFI was first presented in 1982 (7). It is used to calculate the percentage of loss of function in the sciatic nerve of the damaged side when compared to the uninjured side. Both hind legs were pressed with a black ink impregnated stamp on absorbent papers and the SFI, which is a mathematical formula that measures SN malfunction, was measured from the obtained footprints in a 50-cm-long walking corridor (Figure 1). During the placement of the rats in the walking corridor, the walking was repeated until a proper footprint was obtained in the event of distribution of the ink because of urination or walking. 


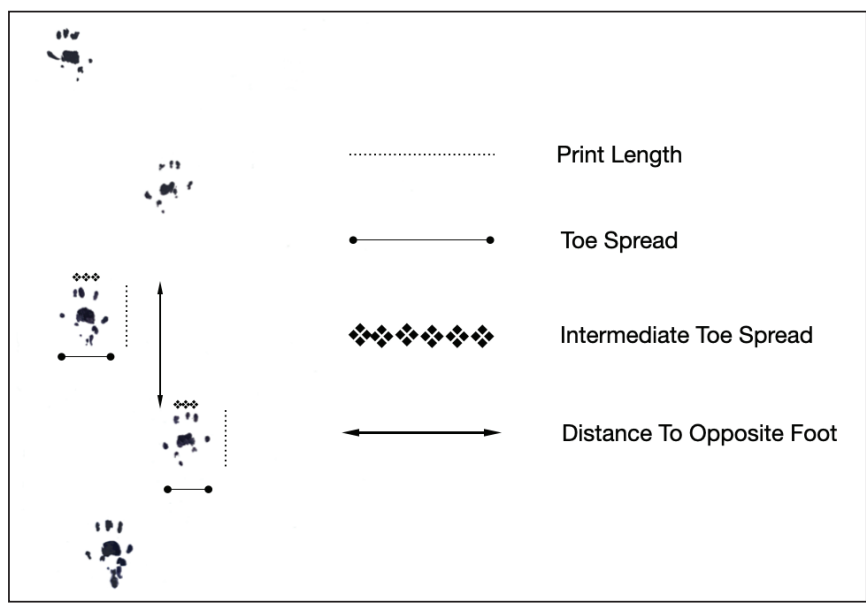

Figure 1: SFI parameters from rat footprints obtained from the walking corridor.

Below is the SFI formula:

$\mathrm{SFI}=[(\mathrm{ETOF}-\mathrm{NTOF}) / \mathrm{NTOF}+(\mathrm{NPL}-\mathrm{EPL}) / \mathrm{EPL}+(\mathrm{ETS}-\mathrm{NTS}) /$ $\mathrm{NTS}+($ EIT $2 \mathrm{NIT}) / \mathrm{NIT})]$ 220/4

Here, TOF is the measurement from the most extreme point of a foot to the other end of the other foot; PL is the footprint length; TS is the toe spread, which is the measurement from first to the fifth toe); and IT is the intermediate toe spread, which is the length from second to the forth toe (7). E refers to the experimental side and $\mathrm{N}$ refers to the normal (uninjured) side. The largest values of the footprint measurements were used for the SFI parameters. The SFI gives a numerical value between -100 and 0 , in which 0 represents normal function of the SN, while -100 represents the full-thickness cut of the SN (Figure 1) (13).

The SFI was measured by the first author for all of the subjects. The rats were numbered and mixed randomly and the person who measured SFI was blind to the treatment procedure that the rats were given. All of the measurements were made blindly to avoid bias. Obtained values were analyzed statistically.

\section{Histopathological Investigation}

After completion of the WTA, SN biopsies were taken from both the injured left $\mathrm{SN}$ and uninjured right $\mathrm{SN}$. SN segments between 10-mm distal and $20-\mathrm{mm}$ proximal to the bifurcation were excised non-traumatically. Damaged and undamaged sciatic nerve tissue samples of the 2 sides were used for the histopathological examinations for AxD, AL (vacuolization), and El. For the histopathological examinations, the hematoxylineosin and toluidine blue staining methods were utilized. AxD, $\mathrm{AL}$, and $\mathrm{El}$ (between $0 \%$ and $100 \%$ ) were analyzed under light microscope with comparison to the uninjured SN segments and the damage levels were scored by dividing them into $10 \%$ intervals.

The second author performed the pathological evaluations for all of the rats. Biopsy materials were numbered and mixed randomly, and the pathologist was blind to the groups and treatment procedures that the rats were given to avoid bias.

\section{Ethics}

Approval for this study was obtained from the Local Ethics Committee of the Experimental Animals Laboratory of the Hospital, (2015-08). All of the rules of law on the protection of experimental animals of The Ministry of Agriculture and Rural Affairs and ethical standards of the 1964 Helsinki declaration were followed.

\section{Statistical Analysis}

The NCSS 2007 software package (Utah, USA) was used. One-way analysis of variance, the Tukey multiple comparison test, Friedman test, and Dunn multiple comparison test were used for the normally distributed variables, subgroups, nonnormally distributed data, and subgroups, respectively. $\mathrm{p}<0.05$ and $\mathrm{p}<0.001$ were accepted as statistically significant and very significant, respectively.

\section{RESULTS}

A comparison of the mean SFI, AxD, and El levels among the groups was conducted. The SFI $(p<0.0001), \operatorname{AxD}(p=0.02)$, and El $(p=0.016)$ levels showed statistically significant differences among all of the groups (Table I).

Table II presents the comparison of the SFI, AxD, and El levels among the groups.

In terms of the $\mathrm{SFI}$, groups $\mathrm{B}_{12}, \mathrm{E}$, and $\mathrm{B}_{12}+\mathrm{E}$ were significantly higher than group $0 \quad(p=0.011, p=0.035, p=0.0001$, respectively). However, groups $B_{12}$ and $E$ were not statistically different. $(p=0.961)$. Group $B_{12}+E$ was statistically significantly higher than groups $B_{12}$ and $E(p=0.048, p=0.015$, respectively).

A comparison of the AxD levels among the groups is seen in Figure 2A-D, where it can be seen that the only significant difference was between groups 0 and $B_{12}+E(p=0.016)$.

Figure $3 A-D$ shows the $A x D$ and El levels in the different rats. Groups $B_{12}$ and $B_{12}+E$ exhibited statistically significantly decreased El levels when compared to group $0(p=0.019$ and $p=0.048$, respectively); however, the mean El levels were not significantly different among the groups ( $p>0.05)$.

\section{DISCUSSION}

$\mathrm{PNI}$ is a challenging health problem with effects that can range from mild discomfort to life-long impairment according to the level of neural involvement (10). PNI can be caused by compression, crush, or transection injuries (17). It can either be acute or chronic, and complete or incomplete (17). Approximately $1.8 \%$ of patients who have had lower extremity trauma suffer from PNI (12). It can cause motor and/ or sensorial dysfunction, social and psychological problems, and loss of work-power (18).

Although repair is slow and often insufficient, the destroyed axons can be regenerated in the PNS, which is known as PNR. $\mathrm{PNI}$ causes endothelial damage and inflammation, which leads to an increase in the permeability of the vessels and edema; thus, neutrophils release chemical mediators, and reactive oxygen species (ROS) and cytokines are produced $(14,16,19)$. 
Albay C. and Kahraman Akkalp A: Peripheral Nerve Healing

Table I: Comparison of Mean SFI Levels, AD and El Among Groups

\begin{tabular}{lccccc}
\hline Mean & Group 0 & Group B12 & Group E & Group B12+E & p \\
\hline SFI & $-36.05 \pm 9.04$ & $-26.88 \pm 5.3$ & $-28.21 \pm 2.6$ & $-19.43 \pm 1.3$ & $\mathbf{0 . 0 0 0 1}$ \\
\hline AxD (\%) & $36.25 \pm 7.44$ & $25 \pm 9.26$ & $26.88 \pm 11$ & $21.88 \pm 7.53$ & $\mathbf{0 . 0 2}$ \\
\hline EI (\%) & $19.38 \pm 13.21$ & $8.13 \pm 0.35$ & $15 \pm 5.35$ & $9.88 \pm 0.35$ & $\mathbf{0 . 0 1 6}$ \\
\hline
\end{tabular}

SFI: Sciatic functional index, AxD: Axonal degeneration, El: Edema/inflammation.

Table II: Tukey Multiple Comparisons of SFI, AxD and EI

\begin{tabular}{llll}
\hline & SFI & AxD (\%) & El (\%) \\
\hline Group 0/B12 & 0.011 & 0.078 & 0.019 \\
\hline Group 0/E & 0.035 & 0.178 & 0.615 \\
\hline Group 0/B12+E & 0.0001 & 0.016 & 0.048 \\
\hline Group B12/E & 0.961 & 0.975 & 0.239 \\
\hline Group B12/ B12+E & 0.048 & 0.896 & 0.96 \\
\hline Group E/ B12+E & 0.015 & 0.68 & 0.487
\end{tabular}

SFI: Sciatic functional index, AxD: Axonal degeneration, El: Edema/inflammation.
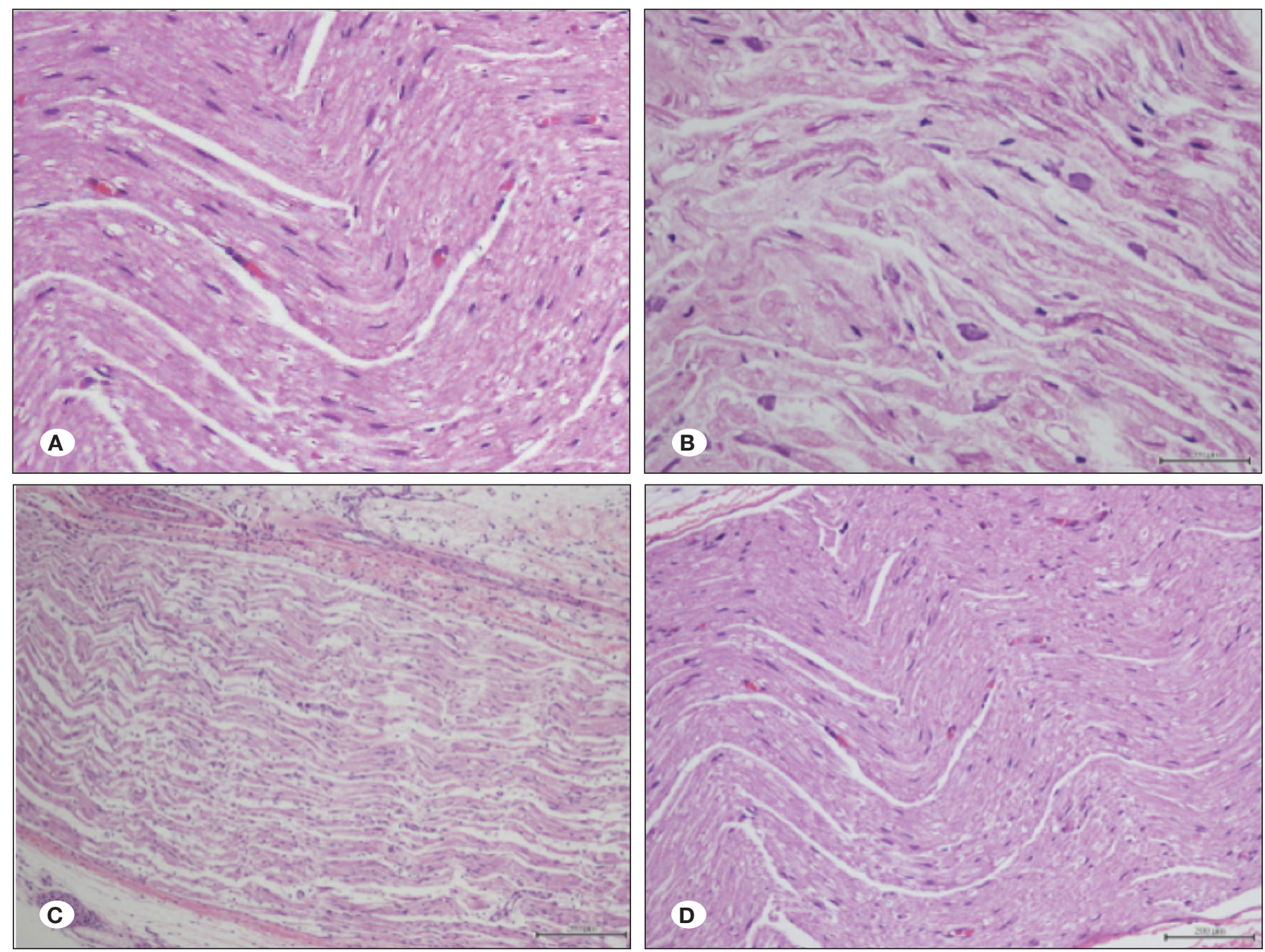

Figure 2: A) Unclamped (normal) sciatic nerve (H\&E, x110); B) AxD, 30\% degeneration, $B_{12}$ group, $\left.H \& E, x 440 ; C\right) ~ A L$ and $A x D, 30 \%$ degeneration, E group, H\&E, x440; and D) minimally damaged nerve preparation, $\mathrm{B}_{12}+\mathrm{E}$ group, H\&E, x220. (H\&E: hematoxylin and eosin). 

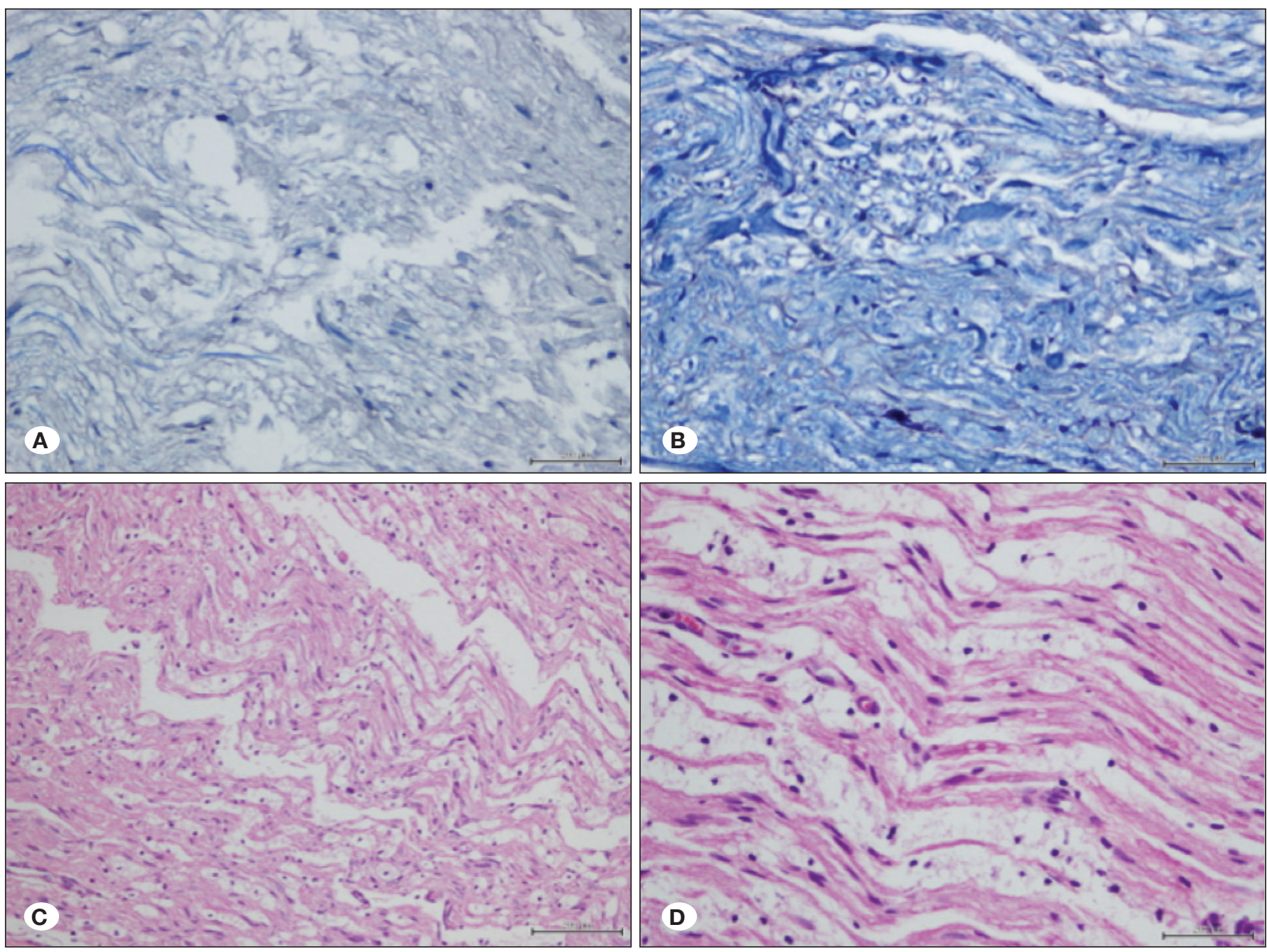

Figure 3: A) AxD and edema, control group, toluidine-blue x440; B) significant AxD and AL in group 0, toluidine-blue x440; C) axon vacuolization and edema H\&E, x220; and D) significant edema and axon vacuolization and inflammation H\&E, x440. (H\&E: hematoxylin and eosin).

As the PN is ensheathed by Schwann cells, which provide trophic support by nerve growth factors (NGFs), the PNS, unlike the central nervous system, has the ability to regenerate (4). The PNR starts from the demyelinated site where contact occurs between the Schwann cells and the axons. The PNR is ensured by the migration of macrophages into the injured area, where contact occurs between the Schwann cells and the axons, and elimination of the demyelinated area takes place. The capability of the PNR is directly related to neurotrophic factors, such as NGFs, which are located on the cell membrane (10).

If the injury causes $20 \%-30 \%$ AxD, the main PNR mechanism is undamaged axon collaterals by the NGFs is; however, if the injury causes more than $90 \%$ AxD, Wallerian degeneration, axonal restoration, and target organ innervation are the 3 main processes of the PNR, and the lack of success in 1 of the 3 processes contributes to inadequate clinical healing (17). The degree of nerve injury, severity of the lesion, length and size of damaged nerve segment, and duration of exposure are the factors that play role in progressive nerve healing. In addition, oxidative stress and the massive production of ROS play a major role in neuronal cell death after PNI $(5,13)$.

Although the mechanisms of PNI and PNR are well-known, fully functional recovery is still limited. There is no complete agreement regarding the treatment protocol for PNI. The main goals of PNI treatment are to accelerate healing and ensure that the healing is nearly complete and to prevent complications and other possible sequelae. PNI can be classified as neuropraxia, axonothymesis, neurotomies, or complete nerve cut (22). Microsurgery is main standard course of therapy in full-thickness nerve cuts; however, the success rate is mostly very limited. As a result, medical supplementations have been attempted in incomplete PNI. As PNR occurs in the first weeks of injury, surgery or medication should be initiated at the earliest possible time (11).

Vitamins have significant roles in nerve transmission (25). Since vitamin $B_{12}$ has antioxidant, antiapoptotic and antinecrotic effects, it has been shown to protect deoxyribonucleic acid, increase muscle cells, and provide nervous tissue formation. 
Vitamin $B_{12}$ induces growth factors and supplies myelin structure and axonal growth $(25,28)$.

Vitamin $E$ is presumed to have a principal role in protecting membrane lipids from lipid peroxidation. Vitamin E derivative supplementation treatment in $\mathrm{PNI}$ in diabetic rats was observed to have decreased oxidative-nithodative stress markers and improved demyelination and nerve blood flow (17). Cisplatin neurotoxicity and diabetic and surgical neuropathies were found to be prevented by vitamin $E$ supplements (27). In addition, vitamin $E$ was found to decrease inflammatory mediators and nociceptive signaling molecules in PNI (17). The combined use of vitamin $E$ and other antioxidant vitamins, such as vitamins $C$ and $A$, was shown to have decreased the oxidative markers of rodents (20). Local vitamin E administration has shown significant improvement and acceleration of PNR, and facilitated the growth and regeneration of injured neurons (21).

In the current study, vitamins $B_{12}$ and $E$, and both together, were found to be effective in the functional recovery of the PNI. The efficacy of vitamin $E$ was found to be as effective as that of vitamin $B_{12}$ in functional recovery, which was in agreement with the literature. According to the SFI, the combination of vitamins $B_{12}$ and $E$ was more effective than that of vitamins $E$ and $B_{12}$ separately. The SFI was higher in the combination treatment when compared with that of vitamin $B_{12}$ alone, which indicated that vitamin $E$ improved the healing effects of vitamin $B_{12}$. In the histopathological evaluation; vitamin $E$ was not effective in the treatment of $A x D$ or El by itself. Although vitamin $B_{12}$ was effective in the treatment of El, it was ineffective in the treatment of AxD by itself. However, the combination of these 2 vitamins decreased both AxD and El. This supported the additive effects of these vitamins, wherein they can reverse neurological injury together.

This study had some limitations, as it did not include an electrophysiological evaluation, such as EMG. However, functional evaluation of the $\mathrm{PN}$ is much more important than electrophysiological evaluations. In literature, it was reported that the use of electrophysiological test was not correlated with functional outcomes, as assessed by the WTA, in rats (8).

\section{CONCLUSION}

Vitamins $B_{12}$ and $E$ were both effective in the functional recovery of PNI. The antiinflammatory healing effects were mainly the result of vitamin $B_{12}$. Neither vitamins $B_{12}$ nor $E$ were effective in axonal regeneration by themselves; however, the combination of these 2 vitamins was effective. The results suggested that vitamin $\mathrm{E}$ was effective in PNI treatment, especially when it was combined with vitamin $B_{12}$. The current study is the only research that has compared the functional and histological recovery outcomes of the separate and combined use of cyanocobalamin and alpha-tocopherol on a nerve injury model. These 2 vitamins are cheap and easy to find, and may be helpful in the treatment of PNI. It is our belief that the combination of these 2 vitamins can be used in the treatment of PNI, especially after future studies have been conducted on humans.

\section{REFERENCES}

1. Albay C, Adanir O, Akkalp AK, Dogan VB, Gulec MA, Beytemur $O$ : Cyanocobalamin and cholecalciferol synergistically improve functional and histopathological nerve healing in experimental rat model. Ideggyogyaszati Szemle 73(3-4):113120,2020

2. Altun I, Kurutas EB: Vitamin B complex and vitamin B12 levels after peripheral nerve injury. Neural Regen Res11(5):842-845, 2016

3. Arikan M, Togral G, Hasturk AE, Horasanli B, Helvacioglu F, Dagdeviren A: Histomorphometric and ultrastructural evaluation of long-term alpha lipoic acid and vitamin B12 use after experimental sciatic nerve injury in rats. Turk Neurosurg 26 (6):944-952, 2016

4. Bagdatoglu C, Saray A, Surucu HS, Ozturk H, Tamer L: Effect of trapidil in ischemia/reperfusion injury of peripheral nerves. Neurosurgery 51(1):212-219; discussion 219-220, 2002

5. Campbell WW: Evaluation and management of peripheral nerve injury. Clin Neurophysiol 119(9):1951-1965, 2008

6. Chabas JF, Stephan D, Marquette T, Garcia S, Lavaut MN, Nguyen C: Cholecalciferol (vitamin D3) improves myelination and recovery after nerve injury. Plos One 8(5):e65034, 2013

7. de Medinaceli L, Freed WJ, Wyatt RJ: An index of the functional condition of rat sciatic nerve based on measurements made from walking tracks. Exp Neurol 77(3):634-643, 1982

8. Dellon AL, Mackinnon SE: Selection of the appropriate parameter to measure neural regeneration. Ann Plast Surg 23:197-202, 1989

9. Dokkaew J, Agthong Z, Suantawee T: Vitamin E reduces sensory neuronal loss and improves Nerve regeneration after sciatic nerve injury. Asian Biomedicine 7(5):649-655, 2013

10. Griffin MF, Malahias M, Hindocha S, Wasim SK: Peripheral nerve injury: Principles for repair and regeneration. Open Orthop J 8:199-203, 2014

11. Hoshida S, Hatano M, Furukawa M, Ito M: Neuroprotective effects of vitamin $\mathrm{E}$ on adult rat motor neurones following facial nerve avulsion. Acta Otolaryngol 129(3):330-336, 2009

12. Huckhagel T, Nüchtern J, Regelsberger J, Gelderblom M, Lefering R, TraumaRegister DGU: Nerve trauma of the lower extremity: evaluation of 60,422 leg injured patients from the TraumaRegister DGU $®$ between 2002 and 2015. Scand J Trauma Resusc Emerg Med 26(1):40, 2018

13. Leker RR, Shohami E: Cerebral ischemia and traumadifferent etiologies yet similar mechanisms: Neuroprotective opportunities. Brain Res Brain Res Rev 39(1):55-73, 2002

14. Li R, Liu Z, Pan Y, Chen L, Zhang Z, Lu L: Peripheral nerve injuries treatment: A systematic review. Cell Biochem Biophys 68:449-54, 2014

15. Li Y, Yao D, Zhang J, Liu B, Zhang L, Feng H, Li B: The effects of epidermal neural crest stem cells on local inflammation microenvironment in the defected sciatic nerve of rats. Front Mol Neurosci 10:133, 2017

16. Lundborg G: A25-year perspective of peripheral nerve surgery: Evolving neuroscientific concepts and clinical significance. J Hand Surg Am 25(3):391-414, 2000 
17. Menorca RM, Fussell TS, Elfar JC: Peripheral nerve trauma: Mechanisms of injury and recovery. Hand Clin 29(3):317-330, 2013

18. Morani AS, Bodhankar SL: Early co-administration of vitamin E acetate and methylcobalamin improves thermal hyperalgesia and motor nerve conduction velocity following sciatic nerve crush injury in rats. Pharmacol Rep 62(2):405-409, 2010

19. Noble M, Tseng KC, Li H, Elfar JC: 4-Aminopyridine as a single agent diagnostic and treatment for severe nerve crush injury. Mil Med 184(1):379-385, 2019

20. Panjwani U, Yadav DK, Kumar A, Singh SB: Effect of vitamin $\mathrm{C}$ and $\mathrm{E}$ supplementation in modulating the peripheral nerve conduction following cold exposure in humans. Int $\mathrm{J}$ Biometeorol 48(2):103-107, 2003

21. Riffel APK, Santos MCQ, de Souza JA, Scheid T, Horst A, Kolberg C, Bello-Klein A, Partata WA: Treatment with ascorbic acid and a-tocopherol modulates oxidative-stress markers in the spinal cord of rats with neuropathic pain. Braz $\mathrm{J}$ Med Biol Res 51(4):e7097, 2018

22. Seddon HJ: Three types of nerve injury. Brain 66:237, 1943

23. Sharma SS, Sayyed SG: Effects of trolox on nerve dysfunction, thermal hyperalgesia and oxidative stress in experimental diabetic neuropathy. Clin Exp Pharmacol Physiol 33(11):10221028, 2006
24. Somay H, Emon ST, Uslu S, Orakdogen M, Meric ZC, Ince $\mathrm{U}$ : The histological effects of ozone therapy on sciatic nerve crush injury in rats. World Neurosurg 105:702-708, 2017

25. Sundem L, Chris Tseng KC, Li H, Ketz J, Noble M, Elfar J: Erythropoietin enhanced recovery after traumatic nerve injury: Myelination and localized effects. J Hand Surg Am 41(10):9991010, 2016

26. Tamaddonfard E, Farshid AA, Maroufi S, Kazemi-Shojaei S, Erfanparast A, Asri-Rezaei S, Taati M, Dabbaghi M, Escort M: Effects of safranal, a constituent of saffron, and vitamin $E$ on nerve functions and histopathology following crush injury of sciatic nerve in rats. Phytomedicine 21(5):717-723, 2014

27. Tiwari V, Kuhad A, Chopra K: Neuroprotective effect of vitamin $\mathrm{E}$ isoforms against chronic alcohol-induced peripheral neurotoxicity: Possible involvement of oxidative-nitrodative stress. Phytother Res 26(11):1738-1745, 2012

28. Wang ZB, Gan Q, Rupert RL, Zeng YM, Song XJ: Thiamine, pyridoxine, cyanocobalamin and their combination inhibit thermal, but not mechanical hyperalgesia in rats with primary sensory neuron injury. Pain 114(1-2):266-277, 2005 